\title{
El conflicto marítimo entre Chile y Bolivia: una mirada desde la responsabilidad colectiva y los derechos humanos
}

\author{
Nicole Selamé Glena*
}

\section{RESUMEN}

Las soluciones que hasta ahora ha dado el Derecho Internacional para resolver el conflicto marítimo entre Chile y Bolivia se han mostrado insuficientes. Este trabajo propone dejar de pensar el conflicto como un problema territorial y bilateral, y enfrentarlo desde la justicia global y los derechos bumanos. El artículo explora los conceptos de responsabilidad nacional, responsabilidad por el resultado y responsabilidad remedial, como contracara de la autonomía y el derecho de autodeterminación. Argumenta que aun cuando Bolivia sea responsable por el resultado de haber perdido el mar, pueden existir buenas razones para que otros agentes de la comunidad internacional -no solamente Chile- tengan la obligación de remediar esta situación, debido a su urgencia y gravedad.

Responsabilidad colectiva - conflicto marítimo Chile/Bolivia - justicia global

\section{The maritime conflict between Chile and Bolivia: a collective responsibility and buman rights approach}

\begin{abstract}
International law has proven itself insufficient to solve the maritime problem between Chile and Bolivia so far. This paper proposes to stop thinking about the conflict as territorial and bilateral and address it from a global justice and human rights' perspective. The paper explores the concepts of national responsibility, outcome responsibility and remedial responsibility, as the flip side of autonomy and self-determination. It discusses that even if Bolivia is outcome-responsible for losing its sea access, there might be good reasons to argue that other agents from the international community - and not only Chile - have the duty to remedy the situation, given its moral urgency.
\end{abstract}

Collective responsibility - Chile/Bolivia maritime conflict - global justice

* Licenciada en Ciencias Jurídicas y Sociales, Universidad de Valparaíso. Doctora en Derecho, Universidad Pompeu Fabra, España. Investigadora del Centro de Investigaciones de Filosofía del Derecho y Derecho Penal, Universidad de Valparaíso. Correo electrónico: nicole.selame@uv.cl.

La autora agradece por el financiamiento a CONICYT + PAI/ concursonacional de apoyo al retorno de investigadores/as desde el extranjero, convocatoria $2014+82140027$.

Artículo recibido el 18.8.2016 y aceptado para su publicación en este número el 5.1.2018. 
$\mathrm{M}$ ás de cien años han transcurrido desde que se inició el conflicto entre Chile y Bolivia y, pese a algunos acercamientos durante el siglo pasado, todo indica que la resolución es difícil y que hoy, más que nunca, nos encontramos lejos de un acuerdo que satisfaga a ambas partes. Pareciera que los argumentos de Bolivia para obtener una salida soberana al Océano Pacífico chocan siempre contra el mismo obstáculo: que ese territorio se perdió en una guerra y que Bolivia es responsable -esto es, debe hacerse cargo- de las consecuencias que se derivan de ello. Efecto de esto es la firma voluntaria entre Bolivia y Chile del Tratado de Paz y Amistad de 1904, en que la primera cede al segundo su única región costera. El Estado boliviano, actuando de manera autónoma y en el ejercicio de su soberanía, habría entrado en un conflicto bélico que perdió, y cuyo fin tuvo que negociar a costa de su integridad territorial. Por tanto, se argumenta, no corresponde a Chile hoy compensar a Bolivia por los resultados de sus decisiones.

Este trabajo busca analizar el problema de Bolivia y de los países sin acceso al mar desde la perspectiva de la justicia global y los derechos humanos. El asunto del que me haré cargo es que algunos países, y en este caso particular Bolivia, se han visto empobrecidos como consecuencia de su falta de acceso marítimo, y la pregunta que intentaré resolver es si existe un deber de darles solución, y si se puede fundamentar este deber en los derechos humanos. Como se trata de obligaciones internacionales es necesario encontrar razones que sean suficientemente universales y urgentes como para que sean aceptadas por todos, y para que su cumplimiento sea exigible por la comunidad internacional.

Lo que intento hacer es determinar si se deben distribuir las cargas que genera el que algunos países no tengan acceso al mar y se vean perjudicados por ello, aun cuando ellos mismos hayan contribuido a este resultado. Si bien puede cuestionarse el alcance de las dificultades asociadas a la mediterraneidad caso a caso, este artículo es una aproximación normativa al problema, por lo que no me detendré en la parte empírica. Explicaré brevemente el caso de Bolivia y las desventajas a las que en general se ven enfrentados los países sin acceso al mar. Me basaré para ello en la abundante bibliografía que existe al respecto y la preocupación manifestada por las Naciones Unidas relativa a los especiales desafíos que sufren los países sin acceso marítimo para alcanzar las metas que, como comunidad internacional, nos hemos planteado como más importantes. El argumento asume que si Bolivia encuentra dificultades para el desarrollo producto de su falta de salida al mar, pueden existir buenas razones para considerar la aplicación de la responsabilidad remedial a este respecto.

El orden que sigue este trabajo es el siguiente. En primer lugar (II) explicaré de manera breve la forma en que Bolivia perdió su acceso marítimo ante Chile y sus constantes intentos reivindicativos. Luego (III) presentaré el contexto general de los países que no tienen acceso al mar y los principales desafíos a los que se enfrentan producto de su mediterraneidad. En el apartado IV y V desarrollaré la teoría de David Miller acerca de responsabilidad colectiva en el contexto de la justicia global, poniendo especial atención en los conceptos responsabilidad por el resultado y responsabilidad remedial. En 
VI y VII aplicaré esta teoría al caso de Bolivia, buscando justificar el acceso marítimo en los derechos humanos. En VIII haré algunos descargos finales.

El término de la Guerra del Pacífico y la posterior firma del Tratado de Paz y Amistad de 1904 significaron para Bolivia no solo la pérdida de parte importante de su territorio, sino también de una cualidad: la de país marítimo. El Tratado de 1904 puso fin al conflicto mediante la cesión por parte de Bolivia a Chile de 400 kilómetros de costa que proyectaban una superficie de 120.000 kilómetros cuadrados correspondientes a la región del Litoral, rica en yacimientos de guano, cobre y salitre. A cambio de ello, Chile le entrega a Bolivia 300.000 libras esterlinas y se compromete a la construcción de un ferrocarril entre Arica y La Paz y a garantizar a Bolivia un libre tránsito desde y hacia el mar.

Desde la firma del tratado Bolivia ha intentado recuperar su acceso marítimo. A diferencia de otras mermas territoriales que sufrió desde su independencia y durante los siglos XIX y XX, esta fue la única que le quitó una cualidad. El conflicto con Paraguay privó a Bolivia de parte del Chaco, pero siguió siendo un país chaqueño; el conflicto con Brasil de parte de la Amazonía, pero siguió siendo un país amazónico. Con Chile, sin embargo, la pérdida de territorio es distinta: al ceder la región del Litoral, Bolivia pierde su cualidad de país marítimo, aunque el ethos de su pueblo siga considerándose ligado al mar. Esto explica que los bolivianos busquen de manera permanente revertir esa pérdida y mantengan viva una simbología asociada al mar y a la reivindicación de un acceso soberano al Océano Pacífico.

Actualmente Bolivia mantiene con Chile una causa pendiente ante la Corte Internacional de Justicia en La Haya, para que esta declare que a) Chile tiene la obligación de negociar con Bolivia para lograr un acuerdo que le garantice un acceso plenamente soberano al Océano Pacífico; b) Chile ha incumplido esa obligación; y c) Chile debe cumplir con la señalada obligación de buena fe, sin demora, formalmente, dentro de un tiempo razonable y de manera efectiva, para garantizar a Bolivia un acceso plenamente soberano al Océano Pacífico ${ }^{1}$. El fundamento de la demanda se encuentra en declaraciones diplomáticas y otros actos oficiales de funcionarios de alto rango chilenos, en que Chile se habría obligado a negociar con Bolivia un acceso soberano al Océano Pacífico. Si bien Chile presentó una excepción preliminar alegando que la Corte carecía de jurisdicción para conocer de la causa, por intentar modificarse un tratado anterior a la celebración del Pacto de Bogotá (el Tratado de 1904), esta fue desestimada por el tribunal, señalando que la fuente de la obligación alegada por Bolivia se encontraría en actos posteriores a la firma del Pacto.

${ }^{1}$ La sentencia de la Corte Internacional de Justicia que falla la excepción preliminar puede encontrarse en http://www.icj-cij.org/docket/files/153/18746.pdf. 
Por la naturaleza de la demanda, la discusión ante la Corte busca determinar si Chile ha contraído o no esa obligación en virtud de los actos de sus representantes y, en caso afirmativo, si la ha incumplido y tiene el deber de cumplirla. Queda fuera de la competencia de la Corte conocer de otros motivos que puedan justificar la entrega a Bolivia de un acceso soberano al mar. Desde esta perspectiva pareciera, entonces, que Bolivia sigue entendiendo el problema como bilateral, y espera que se declare en sede jurisdiccional que Chile debe cumplir la obligación y negociar. La Corte, sin embargo, ya ha señalado que aun cuando acoja la demanda de Bolivia, no podrá predeterminar el resultado de dicha negociación.

Como señalé, este trabajo no busca analizar el conflicto desde el punto de vista planteado por Bolivia ante la Corte Internacional de Justicia, sino que intenta determinar si existen otras razones, desde el punto de vista de los derechos humanos, que permitan afirmar la necesidad de garantizar a Bolivia un acceso al mar o compensarla por ello y en qué medida debe Chile hacerse cargo. Las principales objeciones a la salida marítima, fundadas en la responsabilidad por el resultado y en la inexistencia de obligaciones más allá de nuestras fronteras han hecho sombra a cuestionamientos relacionados con la justicia. En este trabajo intentaré hacerme cargo de la objeción de la responsabilidad, haciendo un análisis del conflicto desde la perspectiva de los derechos humanos y la justicia global. Para ello revisaré la teoría de David Miller acerca de la responsabilidad colectiva, centrándome en la idea de las necesidades básicas como fundamento de la responsabilidad remedial, como alternativa a soluciones como las planteadas por Thomas Pogge o Peter Singer relativas a los deberes de redistribución para los pobres globales.

En la presente sección trataré de dar cuenta de los desafíos especiales a los que se ven enfrentados los países que carecen de acceso marítimo y que nos invitan a pensar el problema como uno de justicia global. Lo que intento argumentar es que, si Bolivia forma parte del grupo de los países acosteros en vías de desarrollo para los que la falta de salida al mar resulta un impedimento en la consecución de las metas que nos hemos propuesto como más importantes, entonces pueden existir buenas razones para considerar que la comunidad internacional debe participar en la solución del problema. Como enfrento el problema desde la perspectiva de los derechos humanos, veré brevemente cuáles son las consecuencias perjudiciales que en general pueden derivarse de la falta de salida al mar, y que nos invitan a considerarlo como un problema de justicia.

La situación de Bolivia no representa un caso aislado. En el mundo existen cuarenta y cuatro países que carecen de acceso marítimo, cifra que aumenta a cuarenta y ocho si incluimos a Nagorno Karabakh, Ossetia del Sur, Kosovo y Transnitria. De estos países, dieciséis están en la lista de las Naciones Unidas de los países en vías de desarrollo (Armenia, Azerbaiyán, Bolivia, Botsuana, Kazajstán, Kirguistán, Mongolia, Paraguay, Macedonia, Moldavia, Sudán del Sur, Suazilandia, Tayikistán, Turkmenistán, Uzbekistán 
y Zimbabue) y otros dieciséis han sido calificados como países menos desarrollados ${ }^{2}$ (Afganistán, Bután, Burkina Faso, Burundi, República Central Africana, Chad, Etiopía, Laos, Lesoto, Malawi, Mali, Nepal, Níger, Ruanda, Uganda y Zambia). Los doce países restantes se encuentran en Europa y, por las condiciones geográficas del continente y de la Unión Europea, no se ven enfrentados a los problemas propios de los países acosteros: ninguna capital está a más de quinientos kilómetros de la costa, cuentan con infraestructura y logística de transporte adecuada, no hay controles fronterizos ni aduaneros, hay paz y estabilidad, existen ríos navegables y en general no es necesario salir de Europa para comerciar, pues lo hacen entre sî́ ${ }^{3}$. Por otra parte, países acosteros de Europa como Luxemburgo, Liechtenstein, Suiza o Mónaco han tenido la oportunidad de dedicarse a actividades financieras para las que el acceso al mar no resulta relevante.

Para los países mediterráneos fuera de Europa, sin embargo, la falta de salida al mar constituye un problema serio. Las mayores dificultades para el desarrollo que sufren estos países encuentran sus causas principalmente en dos factores: la lejanía de los mercados y la dependencia de los países de tránsito. La separación de estos países del mar los sitúa en el extremo de las redes de comercio y transporte, lo que hace que las transacciones de sus productos en el mercado global sean más lentas y más caras, pues deben primero transitar por territorios de otros países para poder llegar a algún puerto donde embarcar sus productos, lo que es costoso en dinero y tiempo. No se trata solamente de la extensión que deben recorrer, sino además que quedan sujetos a condiciones que no dependen de su voluntad y que no pueden controlar, lo que aumenta los riesgos de pérdida, daño y robo. Esto explica por qué la situación de los países sin mar no es igual a la de las regiones interiores de grandes países, que igualmente deben recorrer grandes distancias para llegar al puerto ${ }^{4}$.

La importancia del comercio global hace prácticamente imposible la existencia de Estados autárquicos, y la necesidad de comerciar con el resto del mundo se vuelve onerosa y llena de dificultades para los países acosteros 5 . El mar es el medio por el que viaja la inmensa mayoría de los bienes que se transan en el comercio global: más ecológico y más

${ }^{2}$ Los países menos desarrollados representan el segmento más pobre y débil de la comunidad internacional. Comprenden más del $12 \%$ de la población mundial, pero solo el $2 \%$ del PIB mundial y el $1 \%$ del comercio mundial de bienes. Su bajo desarrollo socioeconómico se caracteriza por capacidades humanas e institucionales débiles, un ingreso bajo y mal distribuido y escasez de recursos. A esto se asocia también inestabilidad política y crisis gubernamentales. Su economía predominantemente agraria y la sola exportación de materias primas los hace vulnerables a crisis externas y se vuelve un círculo vicioso de baja productividad y baja inversión (http://unohrlls.org/about-ldcs/).

${ }^{3}$ Uprety, K. (2006). “The Transit Regime for Landlocked States”, en International Law and Development Perspectives. Washington D.C.: The World Bank y Collier, P. (2007) The Bottom Billion, Oxford: Oxford University Press.

${ }^{4}$ Esto ha sido desarrollado con mayor profundidad en Casal, P. y Selamé, N. (2015). "Sea for the Landlocked: a new Sustainable Development Goal?”, en Journal of Global Ethics, 11:3, pp. 270-279.

${ }^{5}$ Se ha sostenido que puede ser más conveniente para los países acosteros intentar desarrollar una economía autárquica desvinculada del resto del mundo que seguir pagando los altos costos asociados a la mediterraneidad. Véase Friberg, R. y Tinn K. (2009). "Landlocked Countries and Holdup”, en Stockholm School of Economics, disponible en http://www2.hhs.se/personal/tinn/files/trade\%20and\%20holdup.pdf. 
barato que el tránsito terrestre y aéreo, el transporte marítimo monopoliza dicho movimiento. Sin embargo, como he señalado, para llegar al mar estos países se ven forzados a transitar por el territorio de sus países vecinos. Esto significa que la voluntad de los países de tránsito, sumada a la calidad de su infraestructura de transporte, sus procesos administrativos, su paz y estabilidad y sus relaciones diplomáticas con el país acostero van a determinar la facilidad o dificultad con que este último pueda comunicarse con el resto del mundo y enganchar en el comercio global ${ }^{6}$. Debido a que la mayoría de los países acosteros se encuentra en regiones pobres e inestables, por regla general el paso por los países de tránsito resulta complejo ${ }^{7}$.

La distancia promedio a un puerto es de 1.370 kilómetros, mientras que Kazajstán, el país que se encuentra más lejos, está a 3.750 kilómetros, seguido por Kirguistán a 3.600 kilómetros. Estas distancias se vuelven especialmente graves debido a que la mayoría de los países acosteros cuentan con una pésima infraestructura de transporte, y una logística muy deficiente. A modo de ejemplo, solo nueve países tienen más del $50 \%$ de sus carreteras pavimentadas y ocho tienen una cobertura entre el $20 \%$ y el $49 \%$. Esto a su vez se transforma en un círculo vicioso, pues al ser los servicios de transporte tan deficientes hay muy baja demanda, y con ello tampoco hay inversión. La infraestructura inadecuada mantiene la actividad económica en niveles extremadamente bajos, lo que disminuye las ganancias que podrían ser invertidas en servicios sociales ${ }^{8}$. A esto se suma que la inmensa mayoría de estos países no tienen una economía diversificada y son exportadores de materias primas, lo que hace que tengan que exportar grandes volúmenes y la ratio entre los costos de transporte y el precio de los productos sea muy alta 9 .

Las dificultades a las que se ven enfrentados los países acosteros se han traducido en un menor desarrollo. De acuerdo con la UN-OHRLLS, veinte de las cincuenta y cuatro economías de bajos ingresos son Estados acosteros (37\%), mientras solo tres de treinta y cinco son de altos ingresos $(8,5 \%)^{10}$. Como señalé, estos Estados se caracterizan por tener capacidades productivas limitadas y una estructura de exportación no diversificada. Las materias primas agrícolas o mineras equivalen a las tres cuartas partes de sus exportaciones, lo que los hace muy vulnerables a la volatilidad de los precios y los shocks

${ }^{6}$ Collier, P. (2007). The Bottom Billion. Oxford: Oxford University Press: pp. 53-64.

${ }^{7}$ Otro problema significativo de los países acosteros es que se ven excluidos de participar de una cuota del suelo y subsuelo marítimo. Todavía resulta desconocido el valor económico asociado a la explotación de los recursos contenidos en él, y el valor vital de su conservación, y los países mediterráneos no quieren quedar segregados de los beneficios y las decisiones que se tomen al respecto. Véase Tuerk, H. (2015). "Landlocked and Geographically Disadvangaed States", en The Oxford Handbook of the Law of the Sea, Rothwell, D., A. Oude y T. Stephens (eds.), Oxford: Oxford University Press: pp. 325-345.

${ }^{8}$ UN-OHRLLS (2012). Comprehensive Ten-Year Review Conference of the Almaty Programme of Action 2014. Concept Note. Disponible en: http://unohrlls.org/UserFiles/File/LLDC\%20Documents/ALMATY\%20+10/ Concept \%20Note.pdf: pp. 5-6.

${ }^{9}$ UN-OHRLLS (2013a). Landlocked Developing Countries Factsheet 2013. Disponible en: http://unohrlls. org/custom-content/uploads/2013/09/Landlocked-Developing-Countries-Factsheet-2013.pdf.

${ }^{10}$ UN-OHRLLS (2013b). The Development Economics of Landlockedness. Understanding the development costs of being landlocked. Disponible en: http://unohrlls.org/custom-content/uploads/2013/10/Dev-Costs-oflandlockedness.pdf: p. 1. 
externos. Se ven más perjudicados por las crisis alimentarias, el cambio climático, los desastres naturales, la degradación territorial y las crisis financieras y económicas. Esto muchas veces causa un cuantioso patrón de gasto fiscal procíclico, lo que desestabiliza las ya frágiles economías de estos países ${ }^{11}$. En el 2011 las exportaciones de los países acosteros en vías de desarrollo fueron de 1,2\% del total de las exportaciones, y su participación en el comercio mundial fue de $1,15 \%$, lo que muestra su marginalización de la economía global. Todo esto se ha traducido en que los países acosteros crezcan menos que sus vecinos marítimos, que el 40\% de las personas viva con menos de 1,25 dólares al día ${ }^{12}$ y que dieciocho países tengan un PIB per cápita inferior a 1.000 dólares $^{13}$.

Estas dificultades han sido reconocidas por las Naciones Unidas como un obstáculo para el desarrollo. Por ello, en 2003 se realizó la Convención de Almaty, a la que siguió la Declaración de Almaty, en que se busca establecer mejores condiciones para hacer frente a los desafíos de los países que carecen de acceso marítimo. A los diez años de esta convención se buscó complementar estas medidas mediante el plan de acción de Viena del 2014. Tanto las medidas tomadas en Almaty como en Viena tienen que ver con mejorar la infraestructura y logística de transporte y unificar criterios aduaneros que simplifiquen el paso de fronteras. Por otra parte, estas dificultades fueron substancialmente reconocidas en la Millenium Development Declaration que dio lugar a los objetivos de desarrollo del milenio en el 2000 y en la Sustainable Development Declaration que dio lugar a los objetivos de desarrollo sostenible en el 2015, señalando que resulta particularmente complicado que los países acosteros puedan cumplir estas metas por los especiales desafíos a los que se ven enfrentados.

Las soluciones que se han dado hasta ahora por la comunidad internacional son modestas y buscan principalmente acortar los tiempos de tránsito, mejorar la infraestructura y unificar criterios aduaneros. Pienso, sin embargo, que un corredor puede ser una salida más efectiva al reforzar la autonomía de estos países evitando su dependencia de los países vecinos ${ }^{14}$. Como se verá más adelante, la autonomía es un elemento fundamental en la idea de responsabilidad y, así como nos lleva a atribuir consecuencias a las decisiones tomadas colectivamente por las naciones, también debe ser un valor que debemos respetar y promover en el ámbito internacional.

Producto de las dificultades a las que se ven enfrentados, los países acosteros han estado en permanente lucha por obtener una salida marítima, mejores y más seguras

11 UN-OHRLLS (2013b). The Development Economics of Landlockedness. Understanding the development costs of being landlocked. Disponible en: http://unohrlls.org/custom-content/uploads/2013/10/Dev-Costs-oflandlockedness.pdf: p. 15.

${ }^{12}$ UN-OHRLLS (2013b). The Development Economics of Landlockedness. Understanding the development costs of being landlocked. Disponible en: http://unohrlls.org/custom-content/uploads/2013/10/Dev-Costs-oflandlockedness.pdf: p. 27.

13 UN-OHRLLS (2012). Comprehensive Ten-Year Review Conference of the Almaty Programme of Action 2014. Concept Note. Disponible en: http://unohrlls.org/UserFiles/File/LLDC\%20Documents/ALMATY\%20 +10/Concept\%20Note.pdf: pp. 4-5.

${ }^{14}$ Para una visión más profunda sobre esto véase Casal, P. y Selamé, N. (2015). "Sea for the Landlocked: a new Sustainable Development Goal?”, en Journal of Global Ethics, 11:3, pp. 270-279. 
condiciones de tránsito desde y hacia el mar, y participación en la capacidad de protección, exploración y explotación de los océanos y del suelo y subsuelo marítimo. En ocasiones actuando como un bloque con los países geográficamente desaventajados, han buscado negociar con los países de tránsito y obtener de la comunidad internacional normas que aseguren sus derechos. Los Estados acosteros han luchado por un instrumento internacional universal que garantice su derecho, pero la evolución del derecho positivo en la materia se ha gestado a base de tratados específicos y generales que muchas veces han resultado controvertidos y decepcionantes ${ }^{15}$.

Haciendo uso de distintas justificaciones de derecho internacional público como la libertad de los mares, las servidumbres internacionales, la compensación por desigualdades geográficas, etc., no han obtenido más que la declaración de un derecho de libre paso que debe pactarse con el o los países de tránsito por medio de tratados bilaterales o multilaterales. Como señalé anteriormente, por regla general los países sin acceso al mar en vías de desarrollo o menos desarrollados son muy pobres y se encuentran en regiones precarias e inestables. Estas circunstancias hacen que la mayoría de las veces no se encuentren en condiciones demasiado favorables para negociar, pues deben pactar con sus vecinos quienes por regla general están también en condiciones precarias y tienden a aprovechar estas ventajas. Al depender de su voluntad, muchas veces les imponen condiciones gravosas o de explotación, a las que por falta de opciones los países acosteros no se pueden resistir.

\section{IV}

Ante la insuficiencia del derecho internacional para resolver el conflicto, sin normas claras que permitan solucionar controversias entre países acosteros y de tránsito, y sometidos a una negociación asimétrica, la demanda de acceso marítimo de los países que carecen de él resulta un tema complejo. Más aún cuando se habla de acceso soberano y no solamente de mejorar las condiciones de tránsito o de unificar criterios aduaneros. En el caso específico de Bolivia, la principal objeción que se presenta es que el haber perdido el mar fue "su culpa" ${ }^{16}$, en el entendido que fue por sus propias decisiones que tuvo lugar la guerra y la posterior suscripción del Tratado de 1904. De esto se deriva que tiene que cargar con los efectos de esas decisiones, aunque hoy sean perjudiciales, y que debe respetar lo que acordó porque lo hizo en el ejercicio de su autonomía. De otro modo, se argumenta, tendríamos que estar dispuestos a revisar todos los tratados internacional cada vez que un país se encuentre disconforme con sus resultados, lo que intuitivamente parece ir contra el principio de certeza jurídica y restarle seriedad al valor de la autonomía.

${ }^{15}$ Uprety, K. (2006). "The Transit Regime for Landlocked States”, en International Law and Development Perspectives. Washington D.C.: The World Bank: p. 47.

${ }^{16}$ Hago notar acá que con culpa no me refiero a una responsabilidad moral, sino solo a la expresión comúnmente usada para alegar responsabilidad por el resultado. 
Por estos motivos y sin ánimo de desafiar esas intuiciones, para efectos de la argumentación en este trabajo asumiré que Bolivia es responsable por el resultado de haber perdido el acceso al mar y que en principio opera para la comunidad internacional un principio de no intervención. Si las naciones son grupos que tienen la capacidad para buscar sus propias metas y perseguir su destino de acuerdo con sus valores y sus ideas de una vida digna, valiosa o floreciente, no parece adecuado que otros alteren los efectos de sus decisiones de acuerdo con convicciones o prácticas externas. Para mayor claridad explicaré la propuesta de David Miller ${ }^{17}$ acerca de la responsabilidad colectiva en el contexto de la justicia global. De esta manera tendremos mejores herramientas para determinar si Bolivia debe hacerse cargo sola de las consecuencias de su mediterraneidad, o si hay motivos suficientes para que otros agentes también participen de este proceso.

En primer lugar, es necesario especificar qué se entiende por responsabilidad, ya que se trata de un concepto con una pluralidad de significados que puede confundir al lector. En este trabajo voy a hablar de la responsabilidad como el deber de hacernos cargo de ciertos beneficios o cargas, que pueden ser resultado de nuestras acciones o de las de otros, asumiendo las consecuencias respecto de nosotros mismos o bien modificando un estado de cosas respecto de terceros. Si bien es más común hablar de responsabilidad con relación a individuos, en este caso hablaré de responsabilidad colectiva, esto es, la que tienen grupos de individuos y que puede identificarse o atribuirse a un grupo y descender a sus miembros por el solo hecho de pertenecer a él. Esto resulta a veces difícil de aceptar, especialmente por los liberales, porque la agencia se considera un requisito esencial de la responsabilidad, y parece complejo entender que se atribuya consecuencias a alguien solo por el hecho de pertenecer a un grupo. Esta atribución resulta particularmente problemática en el caso de la nación, pues la pertenencia a este grupo por regla general no es voluntaria -característica que nos podría permitir entender más fácilmente que se hagan propios los actos del grupo-, sino que viene determinada por el país en que nacemos o por la sangre de nuestros ascendientes.

Como enuncié, hablaré entonces de responsabilidad nacional como una subespecie de la responsabilidad colectiva. Para Miller ${ }^{18}$, la nación resulta un mejor sujeto que el Estado para asignar o identificar responsabilidades, ya que permite aplicar la responsabilidad a Estados que ya no existen, a naciones que no han actuado por medio del Estado y porque es más fácil traspasar la responsabilidad del grupo a las personas consideradas individualmente. Miller propone dos modelos como tipos ideales a los que las naciones pueden aproximarse, y que justifican que la responsabilidad pase de la nación a los connacionales. Por una parte el modelo del grupo de los que piensan igual (like-minded group model) y, por la otra, el modelo de las prácticas cooperativas (cooperative practice model $)^{19}$. El primero dice relación con grupos de personas que comparten objetivos,

${ }^{17}$ Miller, D. (2007). National Responsibility and Global Justice, Oxford: Oxford University Press.

${ }^{18}$ Miller, D. (2007). National Responsibility and Global Justice, Oxford: Oxford University Press: 110-111).

${ }^{19}$ Miller, D. (2007). National Responsibility and Global Justice, Oxford: Oxford University Press: 114124. Para una crítica a esto véase Pierik, R. (2008). "Collective Responsibility and National Responsibility", en Ethical Perspectives, 19:4, pp. 465-483. 
perspectivas y opiniones, que se reconocen como similares y que cuentan con el apoyo de los demás, aunque no haya uniformidad. El segundo, con grupos que comparten una práctica de la que reciben mutuo beneficio, aunque no piensen igual. Para Miller, si la nación se acerca a esos modelos puede pasarse la responsabilidad a sus ciudadanos. Esto significa que la nación será responsable, pero se podrá exigir a los ciudadanos que vean restringida su libertad para efectos de cargar con los costos de dicha responsabilidad. Si consideramos que la nación es: a) un grupo con una identidad común; b) con una cultura pública común; c) cuyos miembros reconocen obligaciones especiales entre sí; y d) un elemento cuya existencia continua se valora por motivos más allá de los instrumentales, entonces parece fácil verla cercana a estos modelos, cuando sus acciones son expresión de una identidad común o han sido concertadas por canales de acción política ${ }^{20}$.

\section{V}

Dentro de este concepto voy a distinguir dos formas de la responsabilidad colectiva: la responsabilidad por el resultado y la responsabilidad remedial. Esto significa que entenderé que tanto la nación chilena como la boliviana pueden ser responsables de los efectos de las decisiones que han tomado en el curso de la historia, favorables y desfavorables, respecto de sí mismas como de otras naciones. Pero también significa que pueden existir buenas razones para que quien deba encargarse de mejorar un estado de cosas sea un agente distinto de aquel que lo produjo o, lo que es lo mismo, para que no dejemos a las naciones pobres entregadas a su propia suerte. Esto hace que la idea de responsabilidad no repugne a la justicia global, permitiendo que en un mundo interdependiente la responsabilidad se comparta ${ }^{21}$. En este caso, podría significar la obligación de garantizar a Bolivia un acceso al mar, aun cuando haya sido "su culpa" perderlo. Para poder determinar estas responsabilidades es necesario identificarlas, esto es, ver si alguien cumple con las condiciones relevantes para ser responsable, o bien, atribuirlas, es decir, tomar una decisión de entregar los costos o beneficios a un agente, independientemente de si cumple o no con las condiciones, de acuerdo con ciertos criterios. Tanto la responsabilidad por el resultado como la responsabilidad remedial se pueden identificar o atribuir: la identificación puede ser correcta o incorrecta en la medida que se cumplan o no las condiciones relevantes, mientras que la atribución puede estar justificada o no justificada, según los criterios que se apliquen ${ }^{22}$.

Por responsabilidad por el resultado se entiende aquella responsabilidad que mira a los efectos o consecuencias de nuestras acciones. Esto es, tiene que ver con la conexión causal entre determinadas acciones u omisiones y ciertos hechos. Sin embargo, no debe confundirse con la mera causalidad. La causalidad responde a la pregunta de por qué

${ }^{20}$ Miller, D. (2007). National Responsibility and Global Justice, Oxford: Oxford University Press: pp. 124126. Para una explicación de por qué la responsabilidad nacional puede heredarse, véase las páginas 135-161.

${ }^{21}$ Miller, D. (2007). National Responsibility and Global Justice, Oxford: Oxford University Press: p. 112.

${ }^{22}$ Miller, D. (2007). National Responsibility and Global Justice, Oxford: Oxford University Press: p. 84. 
ha ocurrido algo, y porque siempre interviene una sucesión de eventos para que se produzca un resultado, la respuesta dependerá del énfasis que pongamos en determinados hechos. En cambio, la responsabilidad por el resultado tiene que ver con que podamos acreditar a un determinado agente con cierto resultado, que pueden ser pérdidas o ganancias, para sí mismo o para otros. No se requiere intención por parte del agente, y en esto se diferencia de la responsabilidad moral que es más exigente, pero al menos se exige que el resultado deba haber sido previsible ${ }^{23}$. Como acá asumiré que Bolivia es responsable por el resultado de haber perdido el mar, no me detendré en los casos que otros antecedentes causales liberan al agente de responsabilidad, como la enajenación, la manipulación o la coerción.

La responsabilidad remedial, por su parte, tiene que ver con la necesidad de corregir una situación dañosa aun cuando no hayamos sido causa de ella. Es decir, aquí no nos preguntamos por qué se ha producido un determinado resultado, sino quién debe hacerse cargo de él. Si bien muchas veces quien es responsable por el resultado es quien debe corregir la situación, hay casos en que esto no es así y en que se pueden aplicar otros criterios, y casos en que la responsabilidad remedial se puede compartir. Por lo tanto, con la responsabilidad remedial me planteo si existen buenas razones para imponer cargas a determinadas personas o grupos de personas para mejorar un estado de cosas dañoso aun cuando ellos no lo hayan provocado. En el caso que estoy revisando, si puede imponerse a otro u otros países el costo de garantizar a Bolivia un acceso al mar. $\mathrm{Y}$, si es así, nos permite determinar quiénes, de todas las personas o grupos, son los que deben ver afectada su libertad y sus recursos para cumplir con esta tarea.

Las dos variantes de la responsabilidad recién enunciadas buscan reflejar dos aspectos contrastantes de la condición humana. Por una parte, nuestro estar en el mundo como criaturas vulnerables necesitadas de la presencia, recursos y ayuda de otras personas para lograr nuestros objetivos. De esta característica surge una obligación inicialmente difusa de ir en auxilio de quienes lo necesiten que es necesario distribuir de acuerdo con determinados criterios. De esto se encarga la responsabilidad remedial. El otro aspecto de la condición humana relevante a este respecto es el de ser agentes, esto es, autores de nuestra propia vida y de nuestros destinos, los que forjamos por medio de las decisiones que tomamos. De este ejercicio de autonomía surge la obligación de hacerse cargo de las ganancias y pérdidas, tanto para nosotros mismos como para los otros, que se deriven de esas decisiones, y la obligación de los demás de no intervenir ${ }^{24}$.

Por regla general, al hablar de responsabilidad se entiende que nos referimos solo a la responsabilidad por el resultado, esto es, aquella que deriva de nuestra capacidad de agencia. La misma libertad que nos permite actuar es la que nos obliga a hacernos cargo de los resultados de nuestras acciones. De otra forma, no se estaría respetando nuestra autonomía. Como señalé, este es el argumento que principalmente se escucha en contra de la demanda boliviana: si quisieron actuar de una determinada forma, deben hacerse

\footnotetext{
${ }^{23}$ Miller, D. (2007). National Responsibility and Global Justice, Oxford: Oxford University Press: pp. 86-88.

${ }^{24}$ Miller, D. (2007). National Responsibility and Global Justice, Oxford: Oxford University Press: p. 81.
} 
cargo de las consecuencias. Sin embargo, la responsabilidad remedial no debe dejarse de lado pues, como sostuve anteriormente, somos todos en mayor o menor medida personas vulnerables y necesitadas de otros. La responsabilidad remedial es la que nos permite distribuir esa acción externa necesaria para reparar situaciones dañosas y moralmente inaceptables. De este modo, la responsabilidad no se riñe con la justicia global, dándonos la posibilidad de exigir soluciones para los que se encuentren en determinadas situaciones dañosas aun cuando las hayan producido. Es este tipo de responsabilidad el que nos permite distribuir las cargas de mejorar estos escenarios desaventajados de modo que se vuelvan exigibles y su incumplimiento pueda aparejar sanciones.

\section{VI}

Paso ahora a revisar el caso de Bolivia. Considero que, según lo planteado, la aplicación del concepto de responsabilidad remedial permite arrojar luces respecto de cómo enfrentar el conflicto entre Chile y Bolivia desde una perspectiva distinta a la que se ha seguido hasta ahora. Si bien voy a asumir que Bolivia es responsable por el resultado, revisaré brevemente la forma que toma este argumento. Como dije, la responsabilidad por el resultado es el primer elemento que tiende a evaluarse en la discusión del problema entre Chile y Bolivia. La pregunta que se repite es: ¿por qué debería darse una solución a Bolivia si ella misma entró en o generó las condiciones para que se generara la guerra que la dejó sin acceso al mar?

Frente a esto, podría plantear diversas objeciones ${ }^{25}$. Por una parte, podría cuestionarse que el Estado de Bolivia haya efectivamente actuado en representación de la nación, y que por tanto la responsabilidad colectiva pueda pasar a sus miembros considerados individualmente. Si bien no es necesario que haya un régimen democrático para que pueda existir representación, sí debe haber claridad en cuanto a que los actos de los gobernantes al menos coincidan con las creencias y valores de la mayoría ${ }^{26}$. Para determinar esto habría que atender a circunstancias que den cuenta de la forma en que se ejercía el poder y la actitud general frente a las decisiones tomadas. También se puede cuestionar la representación en términos de capacidad. ¿Qué pasa si la mayoría de la población estaba constituida por menores de edad, mujeres sin derechos políticos o miembros de pueblos originarios con insuficiente formación o marginados de la participación? Por otra parte, también es necesario determinar si es posible hacer responsable a las generaciones presentes por los actos cometidos por generaciones pasadas. Esto nos enfrenta a

${ }^{25}$ Miller señala que la extensión de la responsabilidad varía en dos dimensiones. Una es la del control, esto es, el grado en que el pueblo colectivamente podía controlar la dirección de su sociedad. La segunda es la dimensión de la limitación: el grado en que el medio externo provee a la nación de opciones viables en importantes áreas de decisión como la economía y las políticas sociales. Miller, D. (2008). "National Responsibility and Global Justice", en Critical Review of International, Social and Political Philosophy, 11:4, pp. 465-483.

${ }^{26}$ Miller, D. (2007). National Responsibility and Global Justice, Oxford: Oxford University Press: p. 128. 
la dificultad de hacer cargar con el peso de malas decisiones pasadas a niños que nacen en condiciones desaventajadas producto de esas mismas medidas ${ }^{27}$.

Todos estos aspectos son muy difíciles de evaluar desde un punto de vista fáctico y la forma en que se han relatado e interpretado los hechos en ambos países difiere de manera importante. Creo que existen otras disciplinas de las ciencias sociales que cuentan con mejores herramientas que la filosofía del derecho para abordar estos aspectos, por lo que en este trabajo me abstendré de refutar este argumento. Por el contrario, tomaré como supuesto que Bolivia y los actuales miembros de la nación boliviana son responsables por el resultado de haber perdido su salida marítima, y en principio deben hacerse cargo de todas las consecuencias que se sigan de ello, favorables o desfavorables, respecto de sí mismos o de terceros. Asumido esto, intentaré determinar si existen razones suficientes para dejar a Bolivia a su suerte o si hay otros argumentos que permitan afirmar la necesidad de que agentes diferentes de Bolivia y los bolivianos realicen acciones orientadas a modificar la falta de salida al mar.

\section{VII}

En este trabajo intento decir que no se necesita ser cosmopolita para afirmar que existen buenas razones para dar una salida al mar a Bolivia. De lo que se trata es de saber qué condiciones deben darse para que opere la responsabilidad remedial y para que tenga importancia más allá de nuestras fronteras. No es necesario declarar que le debemos a otras naciones lo mismo que a la nuestra ni que las fronteras son moralmente arbitrarias. Es muy importante resaltar esto, porque nos ahorra lidiar con la objeción de que no podemos considerar los problemas de justicia global simplemente como problemas de justicia social en un contexto más amplio. Podemos estar de acuerdo en que nuestras obligaciones son más fuertes para con nuestros connacionales y que hay diferencias relevantes entre la esfera nacional y la internacional, pero eso no nos exime de la obligación de combatir ciertas circunstancias que se presentan como injustas o moralmente inaceptables más allá de nuestro territorio, siempre que su gravedad y urgencia así lo reclamen.

Desde el cosmopolitanismo, podría hablar de la igual obligación de ir en auxilio o de compensar a los pobres del mundo. De acuerdo con Pogge ${ }^{28}$, podría sostener que como las instituciones globales que hemos diseñado son previsiblemente injustas y hay otras alternativas viables que darían un resultado mejor, debemos modificarlas o bien

${ }^{27}$ Para una explicación acerca de cómo justificar la responsabilidad de las generaciones presentes por los actos de generaciones pasadas, véase Miller, D. (2007). National Responsibility and Global Justice, Oxford: Oxford University Press: pp. 135-161.

${ }^{28}$ Véase Pogge, T. (2005). "Severe Poverty as a Violation of Negative Duties", en Ethics and International Affairs, 19:1, pp. 54-83; Pogge, T. (2007). Freedom from Poverty as a Human Right, Oxford: Oxford University Press; Pogge, T. (2008). World Poverty and Human Rights, Cambridge: Polity Press y Pogge, T. (2009). Hacer justicia a la bumanidad. México D.F.: Fondo de Cultura Económica, 2009. 
compensar. Con Peter Singer ${ }^{29}$ podría hablar del igual valor moral de todas las personas y la arbitrariedad de formar parte de una nación u otra. Desde esta perspectiva es indiferente el lugar donde estemos o nuestra nacionalidad para efectos de generar obligaciones de justicia cuando tienen lugar ciertas privaciones. En el primer caso, Pogge pone énfasis en la responsabilidad por el resultado: somos nosotros, los ciudadanos de los países ricos, quienes hemos creado un orden internacional que genera una distribución previsiblemente injusta de los beneficios y cargas que se generan al vivir en comunidad. En el segundo caso, Singer deja de lado la responsabilidad por el resultado y se centra solo en la responsabilidad remedial, señalando que siempre que nos encontremos en condiciones de revertir un mal de cierta gravedad sin un gran costo para nosotros debemos hacerlo, sin preguntarnos por las causas de este fenómeno.

Sin embargo, las objeciones a estas dos formas de cosmopolitanismo pueden ser convincentes. Es muy común que la gente se sienta inclinada a pensar en términos nacionalistas y justifique la redistribución solo respecto de aquellos con quienes comparten un territorio, unos valores, una cultura, una historia y una vida común. Por otra parte, en la esfera doméstica existen instituciones que justifican y permiten la redistribución, lo que es muy difícil de imaginar a nivel global. Por la extensión de este trabajo, no me detendré en ellas, sino que presentaré una alternativa de solución que no requiera de una visión cosmopolita.

En este trabajo busco explicar una forma de argumentar a favor de garantizar a Bolivia una salida marítima que no necesite apelar a una visión cosmopolita. Para ello me valgo de la idea de responsabilidad, y más particularmente de la responsabilidad remedial. Como he señalado, la responsabilidad trabaja con una visión dual del hombre: por una parte, entendiendo que somos sujetos vulnerables e imperfectos que necesitamos de los demás; por la otra, que somos también agentes que actuamos de manera libre y persiguiendo ciertos propósitos, y por tanto debemos hacernos cargo tanto de las cargas como de los beneficios que se generen de nuestras acciones. Esta visión dual es la que nos permite no caer en el error de pensar que los agentes, y en este caso las naciones, pueden siempre mejorar su situación y salir de la pobreza o el sometimiento si toman las decisiones adecuadas aun cuando no cuenten con la participación de otros.

Esto tiene un doble efecto para los países sin mar y el problema que estoy analizando. Por una parte, reconoce que hay casos en que es necesario contar con los otros. Por otro, da un inmenso valor a la autonomía, y una de las cosas que busca una salida marítima es precisamente terminar con la sujeción a la voluntad del país vecino para resguardar la libertad de los Estados acosteros a la hora de perseguir sus fines. Entonces, las dos características principales que constituyen el fundamento de la responsabilidad, la agencia y la carencia, podrían servir para apoyar la necesidad de garantizar un acceso marítimo. Si es necesario hacerse cargo de las consecuencias de nuestros actos porque de otro modo no se respetaría el derecho a autodeterminarse, entonces ese mismo valor

29 Véase Singer, P. (1972). "Famine, Affluence and Mortality", en Philosophy and Public Affairs, 1, pp. 229-243 y Singer, P. (2002). One World: the Ethics of Globalization, New Haven: Yale University Press. 
atribuido a la autonomía es el que nos permite pensar que hay buenas razones para justificar un acceso al mar, frente a otras soluciones más modestas como las planteadas por Viena y Almaty. Y ya que eso es algo que Bolivia no puede garantizarse a sí mismo, la responsabilidad remedial se constituye como una buena herramienta para ello.

Puedo presumir que Miller no diría que hay responsabilidad remedial en el caso de los países acosteros, porque solo la establece en caso de la vulneración de derechos humanos que encuentren su fundamento en necesidades básicas de subsistencia, y la lista que propone es muy restringida. Sin embargo, lo que intento acá es plantear que su teoría podría extenderse a casos como el de los países en vías de desarrollo sin acceso al mar, y al caso de Bolivia en particular, por aplicación de sus criterios de urgencia y universalidad. Esto tomando en cuenta el reconocimiento que se ha hecho por parte de las Naciones Unidas en los Objetivos de Desarrollo del Milenio en el 2000 y en los Objetivos de Desarrollo Sostenible en el 2015, además de las declaraciones de Almaty y Viena de 2003 y 2014, de que los países acosteros se ven enfrentados a desafíos que les hacen mucho más difícil -si no imposible- lograr los objetivos que como comunidad internacional nos hemos planteado como prioritarios y más relevantes, y que buscan precisamente satisfacer estas necesidades básicas de todos los seres humanos.

Voy a explicar este razonamiento. Para que haya responsabilidad remedial es necesario que exista una situación suficientemente urgente y grave que requiera ser subsanada, de modo que justifique imponer obligaciones a terceras personas por perjuicios que ellos no han producido. Tomando en cuenta que por regla general se entiende que estas obligaciones se tienen respecto de personas con las que compartimos cierta historia, valores u objetivos (familia, amigos, comunidad, nación, etc.), parece que se requieren motivos más sólidos para justificar que tengamos obligaciones respecto de personas que están más allá de nuestras fronteras. Para alcanzar la urgencia y gravedad requerida, Miller se vale del concepto de derechos humanos esenciales, que corresponden a un mínimo de demandas razonables que todos tenemos respecto de los demás seres humanos. Debido a que estos derechos imponen deberes a personas en otras partes del mundo, deben tener un alcance universal, de modo que puedan ser aceptados por todos. Por este motivo, no todos los derechos humanos generan responsabilidad remedial ${ }^{30}$.

Para Miller, los derechos que pueden imponer obligaciones de este tipo en outsiders son aquellos que se encuentran fundamentados en una necesidad humana, es decir, aquellos aspectos esenciales de la condición humana que no se eligen y por tanto no dependen de las opciones personales. Pero no basta con que se trate de necesidades que no sean elegidas. Miller agrega además que debe tratarse de necesidades intrínsecas, no instrumentales, cuya satisfacción tiene que ver con evitar que las personas sufran daños. Creo que, si bien el acceso al mar no constituye un derecho humano esencial ni menos una necesidad básica, el consenso internacional respecto de que la falta de salida al mar dificulta el cumplimiento de las metas que hemos elegido como más importantes, permite entender la demanda de Bolivia como un problema de responsabilidad remedial.

\footnotetext{
${ }^{30}$ Miller, D. (2007). National Responsibility and Global Justice, Oxford: Oxford University Press: 178-185.
} 
En este caso particular, esas necesidades básicas podrían fundamentar una obligación de Chile de garantizar una salida a Bolivia por su territorio, sin perjuicio de que exista también una obligación de Bolivia y de la comunidad internacional de compensar a Chile por los costos asociados a ello.

Hay claridad en que no podemos decir que tener acceso al mar sea un derecho humano, y menos una necesidad básica. Sería absurdo sostener que se está vulnerando un derecho humano esencial respecto de la gente que no puede acceder al mar por medio de su propio territorio. La idea de restringir la lista de derechos que sirven para fundamentar la responsabilidad remedial respecto de otros países es precisamente poner énfasis en la urgencia de satisfacer estas necesidades y en su universalidad, con el objetivo de lograr distribuir las obligaciones de una manera eficiente y persuasiva, y terminar con el carácter difuso que por regla general hace que nadie se sienta obligado y que estos deberes no se cumplan. Lo que se busca es acotar los asuntos en que se puede exigir la intervención internacional, para que los casos en que esta obligación se aplique sean exigibles.

Si bien, como he señalado, el acceso al mar no es un derecho humano ni una necesidad humana, las consecuencias que se siguen de la falta de salida marítima en los países en vías de desarrollo sí afectan de manera grave y permanente la protección de los derechos humanos y hacen que las necesidades básicas se vean insatisfechas ${ }^{31}$. De la urgencia y universalidad de las necesidades da cuenta el consenso materializado en que las Naciones Unidas hayan reconocido primero con la declaración de Almaty y la Conferencia de Viena, pero más importante aun, ahora con los Objetivos de Desarrollo del Milenio y los Objetivos de Desarrollo Sostenible que la falta de acceso marítimo es un problema grave del que tiene que hacerse cargo la comunidad internacional lo antes posible.

Me centraré en los Objetivos de Desarrollo Sostenible, que es el actual instrumento en la agenda internacional por el que explícitamente se busca mejorar la vida de las personas globalmente. En su declaración se señala que los diecisiete objetivos propuestos y que deben ser cumplidos antes del 2030 reflejan "el futuro que queremos", esto es, aquello que consideramos como más importante para que todos puedan tener condiciones de vida a lo menos digna. En los documentos elaborados para esta propuesta se reconoce en numerosas ocasiones que para los Estados acosteros es más difícil que para el resto alcanzar las metas que nos proponemos, y que se enfrentan a serias dificultades para lograr estos objetivos como consecuencia de su mediterraneidad. Así, en la introducción a los Objetivos de Desarrollo Sostenible se manifiesta un compromiso a implementar de manera total el Programa de Acción de Almaty para mejorar las condiciones de transporte de los países acosteros pobres y sus países de tránsito y se hace referencia a los especiales desafíos que enfrentan en general respecto de todos los objetivos, y en particular respecto del objetivo 7 (energía asequible y no contaminante), del objetivo 9 (industria,

${ }^{31}$ Como he señalado, este es un trabajo normativo y no empírico, y por tanto no busca desafiar las teorías de que no tener acceso al mar no es un impedimento para el desarrollo. En relación con este problema véase las bibliografía citada para la sección III. 
innovación e infraestructura) y del objetivo 10 (reducción de las desigualdades ${ }^{32}$. Si bien no abogan por un derecho de acceso, sino que plantean medidas más suaves relativas a la disminución de tiempos y mejora en la infraestructura, considero que es más eficiente garantizar un acceso, porque, como he dicho, evita la dependencia de los países de tránsito y fortalece la autonomía de los países acosteros ${ }^{33}$.

\section{VIII}

Algunas reflexiones finales. Como he dicho, este trabajo solo pretende ser una aproximación normativa al problema de Bolivia y no se hace cargo de los asuntos prácticos asociados a la solución del conflicto. Lo que he intentado decir es que, si la falta de salida marítima hace más difícil proteger los derechos humanos que consideramos más importantes, entonces hay buenas razones para pensar que existe una responsabilidad de la comunidad internacional de remediar esta situación. Por el valor que le damos a la autonomía y a la autodeterminación de las naciones, en este trabajo he preferido hablar de una salida soberana frente a otras soluciones indirectas como mejorar las condiciones e infraestructura de transporte y asegurar un tránsito libre y no condicional. Dicho lo anterior, quiero referirme brevemente a dos aspectos a los que se debe atender una vez establecido que hay motivos para aplicar la responsabilidad remedial, esto es, que hay una situación suficientemente urgente y grave para que la comunidad internacional intervenga, aun cuando no haya participado en la producción del resultado. No debemos olvidar que no se trata solo de identificar o atribuir responsabilidad, sino también de poder distribuirla de modo que deje de ser una obligación difusa y pueda exigirse a agentes específicos.

Para estos efectos es necesario, en primer lugar, verificar algunas condiciones. Por una parte, debemos saber si es posible que pueda modificarse la situación dañosa que intentamos enmendar por agencia humana. No puede existir responsabilidad de remediar algo que no se pueda arreglar porque, como dice el aforismo jurídico, a lo imposible nadie está obligado. En este caso específico, debemos atender a si es posible hacer modificaciones que permitan a Bolivia acceder al mar. Ya sea mediante la entrega de un corredor, un enclave, un territorio con soberanía compartida u otras soluciones análogas, no parece algo irrealizable. Segundo, debe poder pedirse a otras personas o naciones, esto es, no puede demandarse algo que solo tenga sentido si se entrega voluntariamente. Miller ejemplifica acá con la obligación de dar amor. Podemos pedir que nos respeten y que

${ }^{32}$ Para un desarrollo en profundidad del acceso al mar como un Objetivo de Desarrollo Sostenible véase Casal, P. y Selamé, N. (2015). "Sea for the Landlocked: a new Sustainable Development Goal?", en Journal of Global Ethics, 11:3, pp. 270-279.

33 No profundizaré en esto ni en la objeción de la fungibilidad (que es mejor compensar a estos países en especie que mediante un acceso), pero un tratamiento más completo puede revisarse en Casal , P. y Selamé, N. (2015). “Sea for the Landlocked: a new Sustainable Development Goal?”, en Journal of Global Ethics, 11:3, pp. 270-279. 
nos traten de manera digna, pero no podemos exigir a nadie que nos quiera, ya que por la naturaleza misma del hecho este no puede ser forzado. En el caso de Bolivia esto no parece ser un impedimento, y prueba de ello es que Chile ha querido, en otros momentos de su historia, sentarse a negociar con Bolivia. Tercero, para que pueda distribuirse la responsabilidad remedial no deben violarse otros derechos. En el caso de Bolivia, esta es una consideración que necesita argumentación adicional, ya que se alega de manera permanente que dar una salida soberana violaría la soberanía y los derechos territoriales de Chile. Sin embargo, Chile es libre de modificar su territorio, mediante cesiones o canjes territoriales, como lo ha hecho en el pasado. Además, pueden invocarse nuevas teorías acerca de la soberanía y los derechos territoriales como las de Arash Abizadeh ${ }^{34}$ o Lea $\mathrm{Ypi}^{35}$, en que la soberanía compartida no implicaría vulneración de derechos. Por último, hay que atender a que el recurso que se disputa no sea escaso, en el entendido que debe ser suficiente para satisfacer las necesidades de todos. Es fácil concluir que este no es el caso, por la inmensa extensión de costa que posee Chile.

Otro aspecto que ha quedado sin tratar son los criterios de distribución de la responsabilidad remedial, es decir, cómo vamos a determinar quién va a ver limitada su libertad y sus recursos para mejorar un estado de cosas en el que puede no haber intervenido. Hasta el momento he hablado de Chile como aquel que debe garantizar a Bolivia una salida marítima, pero no he justificado esta atribución. Por la extensión de este trabajo, solo señalaré que el agente encargado de garantizar la salida no es necesariamente el que debe cargar con todos los costos de esta solución. Puede solicitarse a Chile que garantice el paso por ser la opción más rápida y fácil, pero exigirse a la comunidad internacional que participe de los costos. Además, esto no quita que Bolivia pueda estar obligado a otorgar una compensación territorial o de otro tipo por el acceso.

Para la distribución de estos costos, resulta conveniente la aplicación de la teoría de la conexión de la responsabilidad remedial de David Miller ${ }^{36}$. Esta sostiene que se debe considerar a un agente responsable remedialmente de la situación de otro cuando se encuentre relacionado a él en una o más de las siguientes formas. En primer lugar, cuando el agente sea moralmente responsable de haber causado el daño, es decir, cuando lo haya hecho con intención. En segundo lugar, cuando sea responsable por el resultado, esto es, que pueda ser acreditado con las consecuencias del hecho y que estas hayan sido previsibles al momento de actuar. En tercer lugar, en caso de responsabilidad causal, que consiste en ser parte de la cadena de hechos que desembocaron en un determinado resultado, aunque no haya sido previsible. En cuarto lugar, en aquellos casos en que el agente se beneficie del daño que se ha producido. En quinto lugar, cuando el agente tenga

34 Abizadeh, A. (2012). "The Democratic Legitimacy of Border Coercion: Freedom of Association, Territorial Dominion, and Self-Defence", en American Political Science Association. Disponible en: http://www. researchgate.net/publication/228284021_The_Democratic_Legitimacy_of_Border_Coercion_Freedom_of_ Association_Territorial_Dominion_and_Self-Defence.pdf.

${ }^{35}$ Ypi, L. (2014). “A Permissive Theory of Territorial Rights”, en European Journal of Philosophy, 22:2, pp. 288-312.

${ }^{36}$ Miller, D. (2007). National Responsibility and Global Justice, Oxford: Oxford University Press: pp. 99-109. 
la capacidad de remediar la situación, sin un costo importante para él. Y, por último, cuando exista comunidad entre los agentes, esto es, que se encuentren vinculados por ciertos lazos especiales que pueden ser de muy diversa índole. Si bien no ha sido objeto de este trabajo profundizar en esto, en principio no parece arriesgado decir que Chile participaría de los criterios de responsabilidad causal, beneficio, capacidad y comunidad, y Bolivia a su vez del criterio de responsabilidad por el resultado. La comunidad internacional, por su parte, puede ser también considerada dentro de los criterios de responsabilidad por el resultado' capacidad y comunidad. La forma cómo estos criterios se relacionan entre sí y cómo deberá repartirse la responsabilidad entre estos agentes, sin embargo, deberá quedar para el siguiente trabajo.

\section{BIBLIOGRAFÍA}

Abizadeh, A. (2012). "The Democratic Legitimacy of Border Coercion: Freedom of Association, Territorial Dominion, and Self-Defence", en American Political Science Association. Disponible en: http://www.researchgate.net/publication/228284021_The_Democratic_Legitimacy_of_ Border_Coercion_Freedom_of_Association_Territorial_Dominion_and_Self-Defence.pdf. Casal, P. y Selamé, N. (2015). "Sea for the Landlocked: a new Sustainable Development Goal?", en Journal of Global Ethics, 11:3, pp. 270-279.

Collier, P. (2007). The Bottom Billion, Oxford: Oxford University Press.

Friberg, R. y Tinn, K. (2009). "Landlocked Countries and Holdup”, en Stockbolm School of Economics. Disponible en: http://www2.hhs.se/personal/tinn/files/trade\%20and\%20holdup.pdf, 2009.

Miller, D. (2007). National Responsibility and Global Justice, Oxford: Oxford University Press.

Miller, D. (2008). "National Responsibility and Global Justice", en Critical Review of International Social and Political Philosophy, 11:4, pp. 383-399.

PIERIK, R. (2008). "Collective Responsibility and National Responsibility", en Critical Review of International, Social and Political Philosophy, 11:4, pp. 465-483.

Pogge, T. (2005). "Severe Poverty as a Violation of Negative Duties",en Ethics and International Affairs, 19:1, pp. 54-83.

Pogge, T. (2007). Freedom from Poverty as a Human Right, Oxford: Oxford University Press.

Pogge, T. (2008). World Poverty and Human Rights, Cambridge: Polity Press.

Pogge, T. (2009). Hacer justicia a la humanidad. México D.F.: Fondo de Cultura Económica.

Singer, P. (1972). "Famine, Affluence and Mortality", en Philosophy and Public Affairs, 1, pp. 229-243.

Singer, P. (2002). One World: the Ethics of Globalization, New Haven: Yale University Press.

Tuerk, H. (2015). "Landlocked and Geographically Disadvantaged States", en The Oxford Handbook of the Law of the Sea, Rothwell, D., A. Oude, K. Scott y T. Stephens (eds.), Oxford: Oxford University Press.

UN-OHRLLS (2012). Comprehensive Ten-Year Review Conference of the Almaty Programme of Action 2014. Concept Note. Disponible en: http://unohrlls.org/UserFiles/File/LLDC\%20Documents/ ALMATY\% 20+10/Concept\%20Note.pdf.

UN-OHRLLS (2013a). Landlocked Developing Countries Factsheet 2013. Disponible en: http://unohrlls. org/custom-content/uploads/2013/09/Landlocked-Developing-Countries-Factsheet-2013.pdf.

UN-OHRLLS (2013b). The Development Economics of Landlockedness. Understanding the development costs of being landlocked. Disponible en: http://unohrlls.org/custom-content/uploads/2013/10/ Dev-Costs-of-landlockedness.pdf. 
Uprety, K. (2006). “The Transit Regime for Landlocked States”, en International Law and Development Perspectives. Washington D.C.: The World Bank.

YPI, L. (2014). "A Permissive Theory of Territorial Rights”, en European Journal of Philosophy. 22:2, pp. 288-312. 